\title{
Die Bukowina als eine Insel des „Deutschthums“ im Osten? Deutsche Kulturverbreitung und deren Wahrnehmung in Reiseberichten aus dem 19. Jahrhundert
}

\author{
Alexander Renner \\ Kerngebiet: Österreichische Geschichte \\ eingereicht bei: Martin Rohde, MA \\ eingereicht im: SoSe 2019 \\ Rubrik: Proseminar-Arbeit
}

\begin{abstract}
The Bukovina as an island of "Deutschthum" in the East? The diffusion of German culture and its perception in travel reports from the $19^{\text {th }}$ century

The following seminar paper outlines the description of the Bukovina, a part of the Habsburg Monarchy, in selected travel reports from the 19th century. It explains why the authors of these reports perceived the Bukovina as an island of German culture in Eastern Europe, which was otherwise labelled as barbaric and underdeveloped. It will be shown that the authors'subjective observations are not compatible with up-to-date findings of historical research.
\end{abstract}

\section{Einleitung}

„Bei dem Anblick von Tschernowitz schien uns der ganze europäische Westen nahe vor den Augen gestellt zu sein, und wir glaubten Deutschland deutlich durchzufühlen [...]."1

So beschrieb der Bremer Stadtbibliothekar und Reiseschriftsteller Georg Johann Kohl die Hauptstadt der Bukowina in einem 1841 erschienenen Reisebericht. Nachdem er sich während einer Reise durch das östliche Europa über teils miserable Zustände be-

Georg Johann Kohl, Reisen im Inneren von Russland und Polen, Bd. 3, Dresden-Leipzig 1841, S. 12-13. 
klagte, fühlte er sich in der Bukowina, dem östlichsten Kronland² der Habsburgermonarchie, in den Westen Europas versetzt. Die Projektion des Westens in die Bukowina findet sich nicht nur bei Kohl. Es handelt sich um einen wiederkehrenden Topos. Aber warum ist dies so?

Nachdem die Bukowina 1775 Teil der Monarchie wurde, nahm sich Kaiser Joseph II. ${ }^{3}$ der Aufgabe an, sie an das habsburgische Herrschaftsgebiet anzugliedern. Im Rahmen dreier Ansiedlungsphasen zwischen 1774-1826, des Ausbaus des Schulwesens sowie infrastrukturellen Verbesserungen sollte die Bukowina an die restlichen Länder der Krone angebunden werden. In Reiseberichten aus dem 19. Jahrhundert werden diese Angliederungsprozesse mehrmals mit der Verbreitung deutscher Kultur, Bildung und Sprache, zusammengefasst unter dem Begriff des „Deutschthums”, gleichgesetzt. So machte Joseph Rohrer die Kolonisation des Gebietes durch deutschsprachige Siedler*innen als Grund für die positive Entwicklung des Landes fest. ${ }^{4}$ Karl-Emil Franzos setzte die Errichtung deutschsprachiger Bildungseinrichtungen mit der Verbreitung deutscher Kultur gleich. ${ }^{5}$

An diesen Aussagen setzt die vorliegende Arbeit an. Es wird der Frage nachgegangen, wie die Bukowina in Reiseberichten in Bezug auf die scheinbar starke Präsenz deutscher Kultur, verbreitet durch die Ansiedlung deutschsprachiger Bevölkerung und die Errichtung von Bildungseinrichtungen, beschrieben wurde und wie sich diese subjektiven Beobachtungen mit den Erkenntnissen der neueren Forschung vereinbaren lassen. Die These lautet, dass die Bukowina von den Reiseschriftstellern aufgrund deren Herkunft aus den westlichen, deutschsprachigen Teilen Europas und einer Verbundenheit zur deutschen Kultur durch bewusste Übertreibungen „deutscher" dargestellt wurde, als sie tatsächlich war.

Als Quellen dienen Reiseberichte von Karl-Emil Franzos, Joseph Rohrer und Johann Georg Kohl. Alle Berichte erschienen zwischen 1804 und 1876 und erfuhren zum Zeitpunkt ihrer Erscheinung entsprechende Aufmerksamkeit und Rezeption. Um die eingangs gestellte Frage zu beantworten, werden die Ausführungen der Reiseschriftsteller mit Hilfe von Erkenntnissen der neueren Forschung diskutiert. Dabei kann auf ein breites Spektrum an Literatur zurückgegriffen werden. Kurt Scharr erweist sich als herausragender Kenner der Bukowina. Er behandelte das Kronland in seinen Publikationen „Die Landschaft Bukowina. Das Werden einer Region an der Peripherie 1774-1918“6 und

2 Die Bukowina wurde 1848 zu einem eigenständigen Kronland erhoben: Kurt Scharr, Die Landschaft Bukowina. Das Werden einer Region an der Peripherie 1774-1918, Wien-Köln-Weimar 2010, S. 87.

3 Joseph II. (1741-1790): Sohn von Maria Theresia und Franz Stephan von Lothringen. Ab 1764 römisch-deutscher König, ab 1765 Kaiser des Heiligen Römischen Reiches. Von 1765-1780 Mitregent seiner Mutter, ab 1780 bis zu dessen Tod alleiniger Herrscher: Hans Wagner, Joseph II, in: Neue Deutsche Biographie, Bd. 10, Berlin 1974, S. 617-622.

4 Joseph Rohrer, Bemerkungen auf einer Reise von der türkischen Grenze über die Bukowina durch Ost- und Westgalizien, Schlesien und Mähren nach Wien, Wien 1904, S. 43.

5 Karl-Emil Franzos, Aus Halb-Asien. Culturbilder aus Galizien, der Bukowina, Südrußland und Rumänien, Bd. 1, Leipzig 1876, S. 143-145.

$6 \quad$ Kurt Scharr, Die Landschaft Bukowina. Das Werden einer Region an der Peripherie 1774-1918, Wien-Köln-Weimar 2010. 
"Die Bukowina: Erkundungen einer Kulturlandschaft. Ein Reiseführer"7 Andrei CorbeaHoisie veröffentlichte mit "Die Bukowina und Czernowitz. Hybrider Kulturraum und Faszinosum"8 und "Czernowitz. Der imaginierte Westen im Osten"9 wichtige Beiträge zur Bukowina als Kulturraum.

Im Folgenden wird ein biografischer Überblick über die Autoren geboten, die Entstehung der Reiseberichte kontextualisiert und die Beschreibung der Bukowina im Allgemeinen skizziert. Um die von den Autoren bekräftigte Präsenz des „Deutschthums” greifbar zu machen, werden die von ihnen angeführten Mechanismen zu dessen Verbreitung untersucht. Abschließend werden ausgewählte Beobachtungen der Reiseschriftsteller behandelt.

\section{Autoren und Quellen}

\subsection{Karl-Emil Franzos - "Aus Halb-Asien”}

Karl-Emil Franzos wurde 1848 als Sohn deutsch-assimilierter, ursprünglich sephardischer Juden, in Czortków ${ }^{10}$ geboren. Nach einem Studium der Rechtswissenschaften lebte und arbeitete er ab 1877 als Journalist, Dichter und Autor in Wien. ${ }^{11}$ Bekannt wurde er als Verfasser von Romanen und Erzählungen über das ostjüdische Leben sowie ethnografischer Reisebeschreibungen. ${ }^{12}$ Von 1884 bis 1886 leitete Franzos die "Neue Illustrierte Zeitung" in Wien, ab 1887 die "Deutsche Dichtung" in Berlin. ${ }^{13}$

1876 erschien der erste Band des Werkes „Aus Halb-Asien. Culturbilder aus Galizien, der Bukowina, Südrußland und Rumänien". Franzos behandelte darin eine Reise durch den Osten Europas. Bei dem verwendeten Begriff "Halb-Asien“ handelt es sich um eine rein journalistische Erfindung des Autors. Ursprünglich galt „Halb-Asien“ als Metapher für den Raum zwischen dem, nach Franzos, als gebildet und fortschrittlich geltenden Westen und dem als rückständig bezeichneten Osten Europas, inklusive Asiens. Als Vertreter des deutsch-liberalen Josephinismus setzte sich Franzos für ein „germanisierte[s], freiheitlich regierte[s] Österreich, als Vormacht eines geeinigten Deutschland"14 ein. Dieses Österreich hatte laut ihm die Mission, deutsche Kultur in den Osten zu tragen, um diesem ein kulturelles „Aufranken“ zu ermöglichen. ${ }^{15}$

$7 \quad$ Kurt Scharr, Die Bukowina. Erkundung einer Kulturlandschaft. Ein Reiseführer, Wien-Köln-Weimar 2007.

8 Andrei Corbea-Hoisie, Die Bukowina und Czernowitz. Hybrider Kulturraum und Faszinosum, in: Peter Stachel/ Martina Thomsen (Hrsg.), Zwischen Exotik und Vertrautem. Zum Tourismus in der Habsburgermonarchie und ihren Nachfolgestaaten, Bielefeld 2014, S. 113-122.

9 Andrei Corbea-Hoişie, Czernowitz. Der imaginierte „Westen im Osten“, in: Jacques Le Rider/Moritz Csáky/Monika Sommer (Hrsg.), Transnationale Gedächtnisorte in Zentraleuropa, Innsbruck 2002, S. 79-98.

10 Heutiges Tschortkiw im Westen der Ukraine: JewishGen, Chortkiv, Ukraine, o. D., [https://www.jewishgen.org/ Communities/community.php?usbgn=-1037393], eingesehen 03.04.2020.

11 Österreichisches Biographisches Lexikon, Franzos Karl Emil, o. D. [https://www.biographien.ac.at/oebl/oebl_F/ Franzos_Karl-Emil_1848_1904.xml;internal\&action=hilite.action\&Parameter=franzos*], eingesehen 5.8.2019.

12 Christoph Mick, Reisen nach "Halb-Asien“. Galizien als binnenexotisches Reiseziel, in: Peter Stachel/Martina Thomsen (Hrsg.), Zwischen Exotik und Vertrautem. Zum Tourismus in der Habsburgermonarchie und ihren Nachfolgestaaten, Bielefeld 2014, S. 95-112, hier S. 97.

13 Österreichisches Biographisches Lexikon, Franzos.

14 Karl-Emil Franzos, Mein Erstlingswerk. Die Juden von Barnow, in: Karl-Emil Franzos (Hrsg.), Die Geschichte des Erstlingswerkes, Leipzig 1894, S. 213-284, hier S. 220.

15 Franzos, Aus Halb-Asien, S. 1. 


\subsection{Joseph Rohrer- „Bemerkungen auf einer Reise..."}

Joseph Rohrer wurde 1769 in Wien geboren. Nach Beendigung seines Studiums in Innsbruck nahm er eine Professur für politische Wissenschaften und Statistik an der Universität in Lemberg ${ }^{16}$ an. Bekannt wurde er mit Werken wie "Über die Tiroler" und "Versuch über die Bewohner der österreichischen Monarchie" als Verfasser ethnografischer Berichte über die Bevölkerung der Habsburgermonarchie. In seinen Schriften betonte Rohrer wiederholt, dass diese das Interesse der Leserschaft für den eigenen Staat wecken sollten. Er galt zudem als großer Verehrer Josephs II. ${ }^{17}$

1804 erschienen Rohrers „Bemerkungen auf einer Reise von der türkischen Grenze über die Bukowina durch Ost- und Westgalizien, Schlesien und Mähren nach Wien". Es handelte sich ursprünglich um eine Sammlung von 21 Briefen, die während einer Reise von Suceava ${ }^{18}$ nach Wien zwischen dem 20. November 1802 und dem 15. April 1803 verfasst worden waren. Während zahlreiche statistische Angaben das Bild einer objektiven und wissenschaftlichen Publikation wahren, werden persönliche Wertungen des Autors schnell erkenntlich. Rohrer kritisierte mehrmals wirtschaftliche Fehlentscheidungen, erklärte aber auch die jüdische Bevölkerung zum Feind der christlichen Einwohner*innen von Czernowitz. Diese hätten sich auf Unkosten von christlichen Handelsleuten vermehrt sowie das Fracht- und Fremdenverkehrswesen an sich gerissen. Für Rohrer stellte es eine Gefahr dar, "wenn so wichtige Gegenstände“ in der Hand "Von einer solchen Nation sind". ${ }^{9}$

Rohrers Werke erfreuten sich großer Beliebtheit. 1889 schrieb Karl Hugelmann, ein Wiederabdruck seiner Publikationen wäre „lebhaftem Interesse“ begegnet. Gleichzeitig kritisierte Hugelmann die unzureichende Wissenschaftlichkeit von Rohrers Werken: „Es mag auch zugegeben werden, daß der Charakter strenger Wissenschaftlichkeit vielen der Schriften Rohrer's fehlt."20

\subsection{Johann Georg Kohl - "Reisen im Inneren von Russland und Polen"}

Johann Georg Kohl wurde 1808 in Bremen geboren. Er studierte Rechtswissenschaften in Göttingen, Heidelberg und München, brach das Studium 1830 nach dem Tod seines Vaters aber ab. Mit ersten Ersparnissen finanzierte er Reisen nach Kurland und Livland, ${ }^{21}$ St. Petersburg und in die südlichen Provinzen Russlands. 1838 kehrte Kohl nach Deutschland zurück und veröffentlichte mehrere Bücher, darunter "Reisen im Inneren von Russland und Polen“. In diesem schrieb er unter anderem über eine Reise durch die Bukowina.

16 Heutiges Lwiw im Westen der Ukraine: Sabine Kämper, Lemberg. Die bunte Mitte Europas, in: Geo, o. D., [https:// www.geo.de/reisen/reiseziele/20950-rtkl-lemberg-die-bunte-mitte-europas], eingesehen 03.04.2020.

17 Karl Hugelmann, Rohrer Joseph, in: Allgemeine Deutsche Biografie, Bd. 29, Leipzig 1889, S. 64-68.

18 Stadt im Nordosten Rumäniens: CIVITAS, Suceava, O. D., [https://civitas.eu/city/suceava], eingesehen 03.04.2020.

19 Rohrer, Bemerkungen auf einer Reise, S. 69.

20 Hugelmann, Rohrer, S. 64-66.

21 Historische Landschaften im heutigen Lettland: P. A. Fédor K. Possar, Die russischen Ostsee-Provinzen Kurland, Livaland und Esthland, Stuttgart 1843. 
Kohls Ausführungen über Russland fanden so großen Anklang, dass er sich fortan ganz dem Beruf des Reiseschriftstellers widmete. Es folgten Reisen durch zahlreiche europäische Länder sowie Nordamerika. 1858 kehrte Kohl in seine Heimatstadt Bremen zurück, wo er ab 1863 als Stadtbibliothekar arbeitete. Nach den Recherchen von Andrei Corbea-Hoisie handelte es sich bei Kohl um einen beliebten und sehr gern gelesenen Autoren, der mit seinen Ausführungen maßgeblich den Topos von einem „imaginierten Westen im Osten" prägte.22

\section{Die Beschreibung der Bukowina im Allgemeinen}

Bevor auf ausgewählte Teilaspekte der Reiseberichte eingegangen wird, erfolgt vorerst ein Überblick über allgemeine Beschreibungen der Bukowina.

Als zentrales Merkmal der Reiseberichte ist die von den Autoren forcierte Abgrenzung vom Rest des östlichen Europas zu nennen. Über russländische Gebiete, die Gebiete um die Moldau ${ }^{23}$ und über das im Norden angrenzende Kronland Galizien wurde äußerst negativ berichtet: Während bei Johann Georg Kohl von rauen Völkern südlich der Karpaten zu lesen ist, äußerte sich Franzos über das Kronland Galizien sehr negativ. Von "öde[m] Haideland" gespickt mit "abscheuliche[n] Hüttchen", über "fürchterlich" duftende Städte voller „streitender, schmeichelnder, brüllender, flüsternder, stoßender und zerrender Gestalten, bis hin zu „verwahrlosten Restauration[en] "24 schrieb er. Franzos vertrat das nach Christoph Mick für das 19. Jahrhundert typische Bild eines schmutzigen, armen und rückständigen „Halb-Asiens“.25

In der Bukowina änderte sich das Gemüt der Reiseschriftsteller schlagartig. Rohrer war nach seiner Reise durch die Moldau froh gewesen, wieder auf "österreichischem" Boden angekommen zu sein. ${ }^{26}$ Kohl fühlte sich bei der Ankunft in Czernowitz in den Westen versetzt. ${ }^{27}$ Franzos kam "nach erschwerlicher Fahrt" durch Galizien im "gesegneten Gelände der Bukowina" an. ${ }^{28}$ Auch für die Hauptstadt fand er lobende Worte:

„Der deutsche Geist, dieser gütigste und mächtigste Zauberer unter der Sonne, er - und er allein! - hat dies blühende Städtlein Europa hineingestellt, mitten in die Halbasiatische Kulturwüste."29

Mit dem „deutschen Geist" meinte Franzos einen für ihn spürbaren Einfluss deutscher Kultur, Sprache und Bildung sowie die scheinbare Dominanz deutschsprachiger Bevölkerung.

Corbea-Hoisie, Der imaginierte „Westen im Osten“, S. 87.

Historisches Fürstentum Moldau; heute Teile Rumäniens, der Ukraine und der Republik Moldau: Hans-Christian Maner, Moldau, in: Online-Lexikon zur Kultur und Geschichte der Deutschen im östlichen Europa, 2013, [omelexikon.uni-oldenburg.de/54141.html], eingesehen 03.04.2020. 
Es kann festgehalten werden, dass das Kronland im Gegensatz zu umliegenden Gebieten also positiv beschrieben wurde. Die Autoren machten dabei die Präsenz des "Deutschthums" als maßgeblichen Faktor für den guten Zustand der Bukowina aus. Dieser habe dazu geführt, dass sie alle Nachbarländer „in jeglicher Richtung menschlichen Strebens" überragt hätte. ${ }^{30}$

Bei genauerer Analyse der Quellen ließen sich aber auch negative Aussagen über das Land finden. Rohrer kritisierte besonders die Wirtschaftslage. Exporte in umliegende Gebiete wären zu gering. ${ }^{31}$ Er lobte zwar den Ausbau des Straßen- und Brückensystems unter österreichischer Herrschaft, kritisierte aber zugleich die Missachtung von Wasserstraßen. ${ }^{32}$ Die Kritik an der Bukowina gipfelte in den bereits erwähnten Aussagen gegen die jüdische Bevölkerung des Landes.

Abschließend muss festgehalten werden, dass sich die positive Beschreibung des Kronlandes auf das städtische Milieu, insbesondere die Hauptstadt Czernowitz, beschränkte. Rumänisch und ruthenisch geprägte ländliche Regionen wurden aufgrund der mangelnden Spürbarkeit des deutschen Einflusses deutlich negativer beschrieben und eher "Halb-Asien" zugeordnet. In diesen Regionen habe es lediglich "fleißige Ackersleute" gegeben. ${ }^{33}$

\section{Mechanismen zur Verbreitung deutscher Kultur?}

Die Beschreibung der Bukowina ging für die Reiseschriftsteller also eng mit der Präsenz des „Deutschthums” einher. Für dessen Verbreitung machten die Reiseschriftsteller zwei Mechanismen verantwortlich: Die von Kaiser Joseph II. angeordnete Ansiedlung deutscher Kolonist*innen und die Errichtung deutscher Bildungseinrichtungen. Im Folgenden werden diese beiden Mechanismen sowie deren Auswirkungen untersucht, um so die tatsächliche Ausbreitung deutscher Kultur greifbar zu machen.

\subsection{Die Kolonisation der Bukowina}

Die Bukowina galt Ende des 18. Jahrhunderts als dünn besiedeltes Land. Schätzungen der Bevölkerungszahl variieren je nach Quelle. Während Karl Jakob Freiherr von Enzenberg ${ }^{34}$ eine Zahl von 57.000 schätzte, gab Raimund Friedrich Kaindl ${ }^{35}$ circa einhundert Jahre später etwa 75.000 an. Nach russischen Zählungen lebten im ausgehenden 18. Jahrhundert 68.704 Menschen in der Bukowina. ${ }^{36}$ Insbesondere die Gebiete östlich der Waldkarpaten zeichneten sich durch schwache Siedlungs- und Herrschaftsstrukturen

30 Franzos, Halb-Asien, S. 137.

31 Rohrer, Bemerkungen auf einer Reise, S. 36.

32 Ebd., S. 47.

33 Ebd., S. 70

34 Karl Jakob Freiherr von Enzenberg (1725-1810): Von 1777-1786 oberster Landesverwalter des Hofkriegsrates und als Generalmajor in der Bukowina tätig: Johann Polek, Topographische Beschreibungen der Bukowina mit militärischen Anmerkungen von Major Friedrich von Mieg, in: Jahrbuch des Bukowiner Landesmuseums 5, Czernowitz 1897, S. 3-38.

35 Raimund Friedrich Kaindl (1866-1930): Historiker und Ethnologe, geboren in Czernowitz: Gerhard Grimm, Kaindl Raimund Friedrich, in: Neue Deutsche Biographie, Bd. 11, Berlin 1977, S. 33.

36 Scharr, Die Landschaft Bukowina, S. 183-185. 
aus. Um die Basis für eine gute wirtschaftliche Entwicklung zu legen, die Bindung an das Zentrum der Monarchie zu festigen und die strukturschwache Region qualitativ wie quantitativ aufzuwerten, ließ Kaiser Joseph II. Aufrufe zur Kolonisation veröffentlichen. ${ }^{37}$ Grund, Darlehen für Saatgut, bereitgestellte Geräte und Vieh sollten Anreize für die Ansiedlung darstellen. ${ }^{38}$ Es folgten drei Phasen der Kolonisation. Die erste fand 1774-1786 statt, eine zweite 1786-1790 und die dritte 1790-1826. ${ }^{39}$

Mehrere tausend Familien aus dem südwestdeutschen Raum, aus Schwaben und Böhmen, aber auch Zipser*innen aus Nordungarn ${ }^{40}$ sowie Familien aus Galizien meldeten sich ab 1775 zur angeordneten Ansiedlung. ${ }^{41}$ Die Behörden vor Ort waren mit dem enormen Ansturm überfordert. Interessent*innen mussten oft mehrere Jahre auf die Zuordnung von Land warten. Die erste Kolonisationsphase war zudem von Spannungen zwischen der Militärverwaltung der Bukowina und dem Hofkriegsrat in Wien, welchem die Militärverwaltung unterstellt war, geprägt. ${ }^{42}$ Die Militärverwaltung erachtete die Kolonisation durch Deutschsprachige als zu kostspielig. Sie bevorzugte stattdessen die dauerhafte Ansiedlung lokaler Bevölkerungsgruppen, insbesondere jene von Moldauer*innen. Diese hätten, im Gegensatz zu deutschsprachigen Siedler*innen, eigenes Vieh und Vermögen in die Bukowina mitgebracht, was eine Ansiedlung verhältnismäßig kostengünstig gemacht hätte. 1785 mussten die Pläne des Kaisers vorerst auf Eis gelegt werden. Das Werben um Neusiedler*innen wurde eingestellt, da die Ansiedlung für die Behörden vor Ort organisatorisch und finanziell nicht mehr zu bewältigen war. ${ }^{43}$

Erst zu diesem Zeitpunkt wurden die nötigen Voraussetzungen für eine vom Staat gelenkte Kolonisation geschaffen. Die bestehenden kirchlichen Strukturen der Bukowina wurden aufgelöst: ${ }^{44}$ Klöster wurden aufgehoben, deren Vermögen eingezogen, das Land aus der Metropolie von Jassy ${ }^{45}$ und dem Jurisdiktionsbereich von Konstantinopel herausgelöst. Im Gegenzug kam es zur Errichtung einer eigenständigen Erzdiözese. Mit dem Vermögen der Klöster wurde ein Religionsfond für die griechisch-orthodoxe Kirche geschaffen. ${ }^{46}$ Durch diese Umbaumaßnahmen hatte Wien erstmals die alleinige

37 Kurt Scharr, Die spätneuzeitliche Siedlungstätigkeit in der Bukowina unter österreichischer Herrschaft 17741914. Formung einer Kulturlandschaft durch die Wechselwirkung zwischen Siedlung und dem Entstehen eines modernen Staates, in: 17. Innsbrucker Jahresbericht 2003-2007 der Innsbrucker Geographischen Gesellschaft, Innsbruck 2008, S. 60-77, hier S. 71 .

38 Steffan Luttinger/Ivan Runggaldier, Stadtgenese von Czernowitz mit Blick auf die sozialtopographischen Veränderungen, in: Gunda Barth-Scalmani/Kurt Scharr (Hrsg.), Die Gegenwart des Vergangenen im urbanen Raum Czernowitz-Innsbruck. Projektergebnisse eines gemeinsamen Studienprogrammes der Universitäten Czernowitz und Innsbruck über das kulturelle Erbe im öffentlichen Raum, Innsbruck 2019, S. 15-30, hier S. 16.

39 Scharr, Die Landschaft Bukowina, S. 183

40 Deutschsprachige Bevölkerungsgruppe aus dem historischen Nordungarn; heute Slowakei: K. J. Schröer, Versuch einer Darstellung der deutschen Mundarten des ungarischen Berglandes mit Sprachproben und Erläuterungen, Wien 1864.

41 Luttinger/Runggaldier, Stadtgenese von Czernowitz, S. 16

42 Die Bukowina stand nach dem Anschluss an die Habsburgermonarchie von 1775-1786 unter direkter Militärverwaltung: Scharr, Siedlungstätigkeit, S. 60.

43 Scharr, Die Landschaft Bukowina, S. 188-191.

44 Gemeint sind die kirchlichen Strukturen der moldauischen Orthodoxie.

45 Heutiges lași im Nordosten Rumäniens: Arinda Crăciun, Jassy/laşi, in: Online-Lexikon zur Kultur und Geschichte der Deutschen im östlichen Europa, 2015, [ome-lexikon.uni-oldenburg.de/p32402], eingesehen 03.04.2020. 
Verfügungsgewalt über Grund und Boden der Bukowina, konnte diesen also ohne Mitspracherecht der Kirche vergeben. ${ }^{47}$

Diese Strukturveränderungen führten zu einer zweiten Ansiedlungsphase von 1786 bis 1790. In dieser wurden aber keine neuen Siedler*innen angeworben. Da viele Kolonist*innen der ersten Einwanderungswelle nach wie vor auf die Zuteilung von Land warteten, stand deren Zuweisung im Vordergrund. ${ }^{48}$

Nach dem Tod Josephs II. am 20. Februar 1790 wurde die Kolonisation unter dessen Nachfolger Leopold II. ${ }^{49}$ nicht mehr aktiv vorangetrieben. Trotzdem kam es ab 1790 zu einer dritten Ansiedlungsphase. In dieser Zeit wanderten vor allem Facharbeiter*innen ein. ${ }^{50}$ Wien war in den folgenden Jahren nicht mehr daran interessiert, die Kolonisation staatlich zu lenken. Ab 1814 stand die Eindämmung von Auswanderungsströmen, ausgelöst durch Missernten im selben Jahr, im Vordergrund.

Nur während der ersten Ansiedlungsphase wurden also aktiv Siedler*innen angeworben. Dabei handelte es sich nicht nur um deutschsprachige Kolonist*innen. Sie stammten vielmehr aus den unterschiedlichsten Teilen der Monarchie. Aufgrund dieser Ergebnisse können die tatsächlichen Auswirkungen der Kolonisation in Hinblick auf die damit einhergehende Verbreitung des "Deutschthums" bezweifelt werden: Das von Rohrer angegebene Bevölkerungswachstum von 11.000 auf 33.507 Familien ist nicht alleine auf die Siedlungspolitik Josephs II. zurückzuführen.51 Zu großen Teilen hing es mit der hohen Mobilität der lokalen Bevölkerung zusammen. Diese war häufig von Viehzucht abhängig, lebte dadurch nur selten in dauerhaften Siedlungen und überquerte laufend die Grenze in die Moldau, kehrte aber auch immer wieder in die Bukowina zurück. Dementsprechend schwankte die Bevölkerungszahl permanent. Nachdem das Land 1775 an die Habsburger ging und sich die Befürchtungen einer grausamen Herrschaft nicht bestätigten, kehrten viele der in die Moldau Ausgewanderten zurück. Daher stieg die Bevölkerungszahl zwischen 1774 und 1779 stark an, die staatlich gelenkte Kolonisation machte aber nur einen Teil davon aus. ${ }^{52}$ Die deutschsprachige Bevölkerung stellte zudem zu keinem Zeitpunkt eine Mehrheit in der Bukowina dar. Laut Emil Brix habe es zwar keine absolute Bevölkerungsmehrheiten gegeben, Ruthen*innen und Rumän*innen seien jedoch die am stärksten vertretenen Ethnien gewesen. ${ }^{53}$

47 Scharr, Siedlungstätigkeit, S. 73.

48 Scharr, Die Landschaft Bukowina, S. 193.

49 Leopold II. (1747-1792): Sohn von Maria Theresia und Franz Stephan von Lothringen. Von 1790-1792 Kaiser des Heiligen Römischen Reiches: Adam Wandruszka, Leopold II, in: Neue Deutsche Biographie, Bd. 14, Berlin 1985, S. 260-266.

50 Scharr, Die Landschaft Bukowina, S. 194

51 Rohrer, Bemerkungen auf einer Reise, S. 42.

52 Scharr, Die Landschaft Bukowina, S. 184.

53 Emil Brix, Die Umgangssprachen in Altösterreich zwischen Agitation und Assimilation. Die Sprachenstatistik in den zisleithanischen Volkszählungen 1880-1910, Wien 1982, S. 389. 


\subsection{Die Rolle von Bildungseinrichtungen}

„ICh beginne mit dem Schulwesen. Wie es da 1775 aussah, läßt sich sehr kurz zusammenfassen: es gab auch nicht eine einzige Schule."54 So beschrieb Franzos die Bildungslandschaft der Bukowina vor der österreichischen Herrschaft. Tatsächlich gab es in der Bukowina vor 1775 bereits moldauische Bildungseinrichtungen. Unter österreichischer Herrschaft kam es lediglich zu einer Neugestaltung der Bildungslandschaft. Schulen aus moldauischer Zeit wurden aufgelöst und neue Lehranstalten mit deutscher und rumänischer Unterrichtssprache errichtet..$^{55}$ Insgesamt blieb das Schulwesen in den ersten Jahren der österreichischen Herrschaft aber nur rudimentär. Erst knapp ein Jahrhundert nach der "Annexion" kam es ab 1848 zum flächendeckenden Ausbau des Volksschulwesens. Bis 1850 hatte sich die Zahl der Volksschulen verdreifacht. ${ }^{56}$

Wiederholt lobte Franzos außerdem die deutsche Unterrichtssprache. Tatsächlich war Deutsch aber nur eine der Bildungssprachen. In Grundschulen wurde prinzipiell in der Muttersprache der Schüler*innen unterrichtet. ${ }^{57}$ Die von Wien aus gelenkte Staatlichkeit verfolgte mit dem Schulwesen insbesondere das Ziel der Erzeugung eines kollektiven Österreichbewusstseins. Verbundenheit zur Dynastie als Personifikation eines übernationalen Staates sollte durch das Erzählen einer gemeinsamen Geschichte, mit Hinweisen auf kulturelle Leistungen dieses Staates sowie durch kollektive Erinnerung geschaffen werden. ${ }^{58}$ Die Erzeugung kollektiver Identität war das Ziel. Eine gemeinsame Sprache stand dabei nicht an erster Stelle. Die Regierung in Wien vertrat die Ansicht, dass Loyalität zum Staat auch in anderen Sprachen zum Ausdruck gebracht werden konnte. ${ }^{59}$

Die Ergebnisse zeigen deutlich, dass Schulen nicht zur Verbreitung deutscher Kultur dienten, sondern vielmehr heranwachsende Generationen durch Schaffung einer kollektiven Identität an das Vaterland binden sollten.

\section{Zwischen Darstellung und Realität}

Nachdem die beiden Mechanismen zur (angeblichen) Verbreitung des „Deutschthums” behandelt und relativiert wurden, wird im Folgenden auf ausgewählte Aussagen der Schriftsteller eingegangen.

\footnotetext{
54 Franzos, Aus Halb-Asien, S. 144

55 Caroline Greiderer/Julia Tanaskovic, Identität durch Bildung, Bildungseinrichtungen als Kristallisationspunkt von Identität, in: Gunda Barth-Scalmani/Kurt Scharr (Hrsg.), Die Gegenwart des Vergangenen im urbanen Raum Czernowitz-Innsbruck. Projektergebnisse eines gemeinsamen Studienprogrammes der Universitäten Czernowitz und Innsbruck über das kulturelle Erbe im öffentlichen Raum, Innsbruck 2019, S. 31-44, hier S. 37.

57 Hugo Weczerka, Die „Francisco-Josephina“ in Czernowitz. Eine Universität am Ostrand der Habsburgermonarchie, in: Wolfgang Dahmen/Johannes Kramer/Victoria Popovici (Hrsg.), Gelebte Mulikulturalität. Czernowitz und die Bukowina, Frankfurt am Main 2010, S. 67-86, hier S. 71.

58 Greiderer/Tanaskovic, Identität durch Bildung, S. 33-34.

59 Ebd., S. 44
} 


\subsection{Die Dominanz der deutschen Sprache?}

Wie bereits mehrfach darauf hingewiesen, war das „Deutschthum“ für Franzos das "herrschende Element des Landes".60 Besonders ersichtlich sei dieser Umstand an der Dominanz der deutschen Sprache gewesen. Diese sei einerseits die offizielle Amtsund Schulsprache, andererseits aber ebenso die Sprache der Gebildeten sowie jene der Kunst gewesen. ${ }^{61}$ Auch Kohl sah in der deutschen Sprache das Kennzeichen für die Verbreitung deutscher Kultur. ${ }^{62}$

Diese Beschreibungen legen eine tatsächliche Dominanz des Deutschen nahe. Volkszählungen und die damit verbundene Erhebung von Umgangssprachen der Bevölkerung des Kronlandes scheinen diese Vermutung auf den ersten Blick zu bestätigen. Bei der Volkszählung 1880 bekannten sich von den 568.453 Einwohner*innen der Bukowina 19,14 Prozent zur deutschen Sprache, 3,21 Prozent zur polnischen, 42,16 Prozent zur ruthenischen, 33,43 Prozent zur rumänischen und 1,74 Prozent zur magyarischen Sprache. Die restlichen 0,32 Prozent entfielen auf andere. ${ }^{63}$ Von den 54.171 Einwohner*innen von Czernowitz gaben 52,4 Prozent Deutsch als Umgangssprache an. ${ }^{64}$

Die in den Volkszählungen der cisleithanischen Reichshälfte erhobenen Umgangssprachen sind jedoch nicht mit der Muttersprache und schon gar nicht mit der Nationalität der Bevölkerung gleichzusetzen. Als Umgangssprache konnten die Befragten bei einer Volkszählung nur jene Sprache angeben, mit der sie sich im Moment am meisten identifizierten, unabhängig von der eigentlichen Nationalität. Nach Emil Brix war eine Umgangssprache ein „sozial bedingtes Moment“65

Anhand einer Analyse der Volkszählung von 1910 wird eine weitere Problematik ersichtlich. Von den etwa 800.000 Einwohner*innen der Bukowina gaben circa zwanzig Prozent Deutsch als Umgangssprache an. Dieser Anteil setzte sich aus 72.000 Christ*innen $^{*}$ und 96.000 Jüdinnen und Juden zusammen. Jiddisch stand nicht als Umgangssprache zur Wahl. Aufgrund der politischen Verhältnisse wählten große Teile der jüdischen Bevölkerung, egal woher sie kamen, Deutsch als Umgangssprache. Dies sei nach Brix vor allem ein Zeichen der starken politischen Stellung der Deutschsprachigen gewesen. ${ }^{66}$ Dieser Umstand zeigt aber auch, dass deutschsprachig in diesem Zusammenhang nicht zwangsweise mit deutscher Nationalität gleichzusetzen ist.

Die Präsenz der deutschen Sprache, insbesondere ihre Verbreitung im städtischen Milieu, kann nicht geleugnet werden. Besonders die Tatsache, dass Deutsch als offizielle Verwaltungssprache galt, unterstreicht die große Bedeutung. Ländliche Gebiete wiesen aber einen viel geringeren Prozentsatz auf. Im Norden war beispielsweise die ruthenische Sprache vorherrschend. ${ }^{67}$

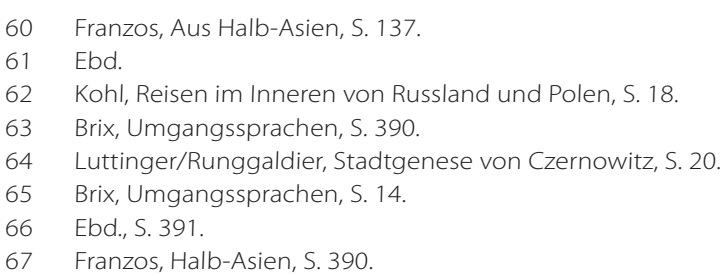




\subsection{Die Franz-Josephs-Universität als eine Bastion des "Deutschthums"?}

Der Czernowitzer Franz-Josephs-Universität wurde in den Ausführungen von Franzos eine besondere Bedeutung zuteil. Die Jubiläumsfeier der hundertjährigen Zugehörigkeit der Bukowina zu Österreich sowie die gleichzeitige Eröffnung der Universität am 4. Oktober 1875 waren die ausschlaggebenden Gründe für dessen Reise gewesen. Dementsprechende Beachtung fand die Hochschule im Kapitel „Ein Culturfest”.

Zwar sei die Hochschule aus einem echten Bedürfnis heraus entstanden, war aber auch eine "friedliche Schutzwehr für das bedrohte deutsche Volksthum im Osten"68. Sie galt für Franzos als „Erhalterin und Mehrerin der deutschen Kraft im Osten" ${ }^{\prime 69}$. Er schrieb der Hochschule also eine vermittelnde, gar missionarische Rolle zu. Das "Deutschthum” sollte durch sie erhalten, verbreitet und geschützt werden. Es stellt sich nun die Frage, welche Bedeutung die Franz-Josephs-Universität tatsächlich hatte.

Der Wunsch nach einer Hochschule entstand in der Bukowina aus einer ganz simplen Problematik heraus. Die Distanz zu Universitäten mit im Land geläufiger Bildungssprache war zu groß.70 Dem Land fehlte es zudem an guten, mehrsprachigen Fachkräften. Eine Ausbildungsstätte auf hohem wissenschaftlichen Niveau für die orthodoxe Geistlichkeit existierte ebenso nicht. Deswegen wurde 1872 ein Antrag zur Errichtung einer Hochschule, eingebracht von Konstantin Tomaszczuk, ${ }^{71}$ im Bukowiner Landtag angenommen und nach Wien weitergeleitet.72 Dieser wurde am 20. März 1875 vom österreichischen Parlament angenommen und am 31. März 1875 vom Kaiser gebilligt.73

Bezüglich der Bedeutung dieser Hochschule müssen die Aussagen von Franzos wiederum relativiert werden. Einerseits galt sie als die kleinste Hochschule der Monarchie mit einem damit einhergehenden geringen Einflussbereich. In den ersten 15 Jahren schwankte die Zahl der Studierenden zwischen 177 und 285. Zu Beginn des 20. Jahrhunderts hatte die Universität nur knapp mehr als fünfhundert Studierende. ${ }^{74}$ Erst 1909/10 übersprang sie die Tausendergrenze. ${ }^{75}$ Andererseits war das Ansehen der "Francisco-Josephina” im Vergleich zu anderen Hochschulen der cisleithanischen Reichshälfte deutlich geringer. ${ }^{76}$

68 Franzos, Aus Halb-Asien, S. 156

69 Ebd., S. 157

70 Die nächstgelegenen Universitäten wären in Wien, Graz und die deutschsprachige Universität in Prag gewesen: Weczerka, Die "Francisco-Josephina", S. 67-86.

71 Dr. Konstantin Tomaszcuk (1840-1889): Jurist und Politiker der deutschliberalen Partei aus Czernowitz. Ab 1871 im bukowiner Landtag, ab 1872 im bukowiner Gemeinderat. Ab 1875 erster Rektor der Universität Czernowitz: Österreichisches Biographisches Lexikon, Tomaszczuk (Tomaszuk) Konstantin (Constantin), o. D., [https://www. biographien.ac.at/oebl/oebl_T/Tomaszczuk_Konstantin_1840_1889.xml], eingesehen 4.2.2020.

72 Weczerka, Die „Francisco-Josephina“, S. 73.

73 Ebd., S. 74.

74 Zum Vergleich Zahlen der Studierenden aus dem Jahr 1899: 6000 Studierende in Wien, 3000 an der tschechischen Universität in Prag, 2000 in Lemberg, 1500 in Graz, 1300 in Krakau, 1300 an der deutschsprachigen Universität in Prag und 1000 in Innsbruck, der zweitkleinsten Universität der Monarchie: Weczerka, Die „Francisco-Josephina“, S. 82.

76 Weczerka, Die „Francisco-Josephina“, S. 80. 
Nichtsdestotrotz war die Universität stark vom Typus der deutschen Hochschule geprägt. Bei Eröffnung der „Francisco-Josephina” waren 14 der insgesamt 28 Lehrstuhlinhaber Deutsche, zehn Rumänen und vier Ruthenen. ${ }^{77}$ Während der gesamten österreichischen Zeit waren 86 der 127 Lehrpersonen Deutsche, ebenso 22 der 44 Rektoren. ${ }^{78}$ Die meisten nichtdeutschen Lehrkräfte, darunter Rumänen, Ruthenen, Slowenen oder Tschechen, wurden von anderen Universitäten der cisleithanischen Reichshälfte oder von deutschen Hochschulen rekrutiert. ${ }^{79}$ Zwar kann die deutsche Prägung der Hochschule nicht bestritten werden, Franzos Zuschreibungen erweisen sich bei genauerer Betrachtung aber als nicht haltbar.

\subsection{Ethnische Diversität und friedliches Zusammenleben?}

Franzos betonte an mehreren Stellen seines Reiseberichtes die ethnische und konfessionelle Vielfalt. Das „Deutschthum“ habe diese friedliche Koexistenz erst ermöglicht. ${ }^{80}$ Für Rohrer war ethnische Diversität eher eine Tatsache, die von der Bevölkerung akzeptiert werden musste. Einen expliziten Willen zum friedlichen Zusammenleben erkannte er nicht. ${ }^{81}$

In der Forschung herrscht prinzipiell Einigkeit über die friedliche Koexistenz der zahlreichen Ethnien der Bukowina. Brix merkte an, dass das Land über keine Bevölkerungsmehrheiten verfügte. ${ }^{82}$ Peter Rychlo kam zu demselben Ergebnis. Keine Ethnie hätte eine absolute Mehrheit aufweisen können, vielmehr habe es sich um eine Ansammlung von Minderheiten gehandelt. ${ }^{83}$ Lediglich der Terminus "Zusammenleben“ erwies sich innerhalb der Forschung als umstritten. Kurt Scharr spricht von „Nebeneinanderleben“: Es hätte sich „weniger um [ein] Leben der verschiedenen Nationen miteinander, sondern vielmehr [um] ein weitgehend von Toleranz - trotz aller Konflikte - geprägtes Leben nebeneinander" gehandelt. ${ }^{84}$

Der Grundstein für die friedliche Koexistenz wurde durch eine tolerante Nationalitätenpolitik gelegt. Die Nationalitätenfrage spielte in der Bukowina deswegen auch nach den Revolutionen von 1848 keine große Rolle. Zwar bildeten sich ab 1849 ein eigenes Landesbewusstsein und nationale Strömungen heraus, die fehlende Dominanz einer Ethnie führte aber weiterhin zu konsensorientierter Zusammenarbeit. Als Beispiel dafür kann der Bukowiner Ausgleich von 1910 genannt werden, welcher von Vertretern aller Ethnien des Landes ausgearbeitet wurde. ${ }^{85}$

77 Weczerka, Die „Francisco-Josephina“, S. 77.

78 Mit „Deutsch“ ist an dieser Stelle stets die Nationalität gemeint.

79 Weczerka, Die „Francisco-Josephina“, S. 80-81.

80 Franzos, Aus Halb-Asien, S. 136-137.

81 Rohrer, Bemerkungen auf einer Reise, S. 43.

82 Brix, Umgangssprachen, S. 389.

83 Peter Rychlo, Czernowitz als geistige Lebensform. Die Stadt und ihre Kultur, in: Helmut Braun (Hrsg.), Die Geschichte einer untergegangenen Kulturmetropole, Berlin 2006, S. 7-30, hier S. 28-29.

84 Scharr, Erkundung einer Kulturlandschaft, S. 120.

85 Politischer Ausgleich zwischen den in der Bukowina lebenden Ethnien in der Frage der Landesselbstverwaltungsorgane und der Vertretung im Landtag: Haus der Bayrischen Geschichte, o. D., [http:// www.hdbg.de/integration/de/b/gross/h-bukowinatext.htm], eingesehen 6.2.2020. 
Trotzdem gab es im 19. Jahrhundert Nationalitätenkonflikte zwischen Rumän*innen und Ruthen*innen. Durch die große Zuwanderung von Ruthen*innen fühlten sich die Bukowiner Rumän*innen als vermeintliche Minderheit im Land gefährdet. Als Konsequenz förderte der griechisch-orthodoxe Religionsfond exklusiv die rumänische Bevölkerung. Die Ruthen*innen reagierten mit privaten Bildungs- und Spracheinrichtungen. ${ }^{86}$ Zu einer Eskalation der Nationalitätenkonflikte kam es in der Bukowina erst mit Ende des Ersten Weltkrieges und dem Zerfall der Monarchie ab 1918.

\section{Resümee}

Ausgangspunkt war die Frage, wie die Bukowina in Reiseberichten aus dem 19. Jahrhundert beschrieben wurde. Anhand von drei Reiseberichten konnte eine allgemeine Beschreibung des Kronlandes geboten werden. Als wesentliches Merkmal stellte sich die Abgrenzung vom Rest "Halb-Asiens" heraus. Während der Osten Europas als unkultiviert, ungebildet und fremdartig galt, stellte das östlichste Kronland der Monarchie, insbesondere dessen Hauptstadt Czernowitz, das Abbild des Westens im Osten dar. An mehreren Stellen wurde deutlich, dass ein scheinbar stark spürbarer Einfluss deutscher Kultur, Bildung und Sprache als Ursache für diese Wahrnehmung galt. Die Ansiedlung deutscher Kolonist*innen sowie der Ausbau des Schulwesens unter Kaiser Joseph II. ermöglichten laut Franzos, Rohrer und Kohl die Verbreitung des „Deutschthums”. Es konnte bestätigt werden, dass diese Aktionen zwar stattfanden, deren Relevanz, Bedeutung und Folgen wurden jedoch klar relativiert.

Die Reiseschriftsteller bewiesen die Verbreitung deutscher Kultur wiederholt mit einer scheinbaren Dominanz der deutschen Sprache. Durch eine Analyse der Erhebung der Umgangssprachen konnte diese angebliche Dominanz differenziert betrachtet werden. Auch die Rolle der von Franzos gelobten Franz-Josephs-Universität als Bastion deutscher Kultur und Bildung wurde kritisch diskutiert. Die Universität entstand aus einem realen Bedürfnis und mit dem Ziel, Kultur zu verbreiten.

Die Aussagen über die friedliche Koexistenz aller Ethnien der Bukowina konnten bestätigt werden. Zwar gab es zwischen Ruthen*innen und Rumän*innen immer wieder kleinere Konflikte, eine tolerante Nationalitätenpolitik förderte aber insgesamt die friedliche Gesinnung zwischen den ethnischen Gruppen.

Diese Ergebnisse zeigen, dass es an mehreren Stellen zu einer deutlichen Übertreibung der Reiseschriftsteller bezüglich der tatsächlichen Verbreitung des "Deutschthums" kam. Die eingangs aufgestellte These kann also zu großen Teilen bestätigt werden. Die aufgezeigte positive Beschreibung der Bukowina beschränkte sich vor allem auf das städtische Milieu. Rumänisch und ruthenisch dominierte ländliche Regionen zählten die Reiseschriftsteller eher zu "Halb-Asien“. Insgesamt hing die Begeisterung der Autoren für die Bukowina stark von der Spürbarkeit des "Deutschthums" ab. 


\section{Literatur und Quellen}

Brix, Emil, Die Umgangssprachen in Altösterreich zwischen Agitation und Assimilation. Die Sprachenstatistik in den zisleithanischen Volkszählungen 1880 bis 1910, Wien 1982.

CIVITAS, Suceava, o. D., [https://civitas.eu/city/suceava], eingesehen 03.04.2020.

Corbea-Hoişie, Andrei, Czernowitz. Der imaginierte „Westen im Osten“, in: Jacques Le Rider/ Moritz Csáky/ Monika Sommer (Hrsg.), Transnationale Gedächtnisorte in Zentraleuropa, Innsbruck 2002, S. 79-98.

Ders., Die Bukowina und Czernowitz. Hybrider Kulturraum und Faszinosum, in: Peter Stachel/Martina Thomsen (Hrsg.), Zwischen Exotik und Vertrautem. Zum Tourismus in der Habsburgermonarchie und ihren Nachfolgestaaten, Bielefeld 2014, S. 113-122.

Crăciun, Arinda, Jassy/laşi, in: Online-Lexikon zur Kultur und Geschichte der Deutschen im östlichen Europa, 2015, [ome-lexikon.uni-oldenburg.de/p32402], eingesehen 03.04.2020.

Franzos, Karl-Emil, Aus Halb-Asien. Culturbilder aus Galizien, der Bukowina, Südrußland und Rumänien, Bd. 1, Leipzig 1876.

Franzos, Karl-Emil, Mein Erstlingswerk. Die Juden von Barnow, in: Karl-Emil Franzos (Hrsg.) Die Geschichte des Erstlingswerkes, Leipzig 1894, S. 213-284.

Greiderer, Caroline/Tanaskovic, Julia, Identität durch Bildung. Bildungseinrichtungen als Kristallisationspunkt von Identität, in: Gunda Barth-Scalmani/Kurt Scharr (Hrsg.), Die Gegenwart des Vergangenen im urbanen Raum Czernowitz-Innsbruck. Projektergebnisse eines gemeinsamen Studierendenprogrammes der Universitäten Czernowitz und Innsbruck über das kulturelle Erbe im öffentlichen Raum, Innsbruck 2019, S. 31-44.

Grimm, Gerhard, Kaindl Raimund Friedrich, in: Neue Deutsche Biographie, Bd. 11, Berlin 1977, S. 33.

Haus der Bayrischen Geschichte, o. D., [http://www.hdbg.de/integration/de/b/gross/hbukowinatext.htm], eingesehen 06.02.2020

Hugelmann, Karl, Rohrer Joseph, in: Allgemeine Deutsche Biographie, Bd. 29, Leipzig 1889, S. 64-68.

JewishGen, Chortkiv, Ukraine, O. D., [https://www.jewishgen.org/Communities/community.php?usbgn=-1037393], eingesehen 03.04.2020.

Kämper, Sabine, Lemberg. Die bunte Mitte Europas, in: Geo, o. D., [https://www. geo.de/reisen/reiseziele/20950-rtkl-lemberg-die-bunte-mitte-europas], eingesehen 03.04.2020.

Kohl, Johann Georg, Reisen im Inneren von Russland und Polen, Bd. 3, Dresden-Leipzig 1841.

Luttinger, Stefan/Runggaldier, Ivan, Stadtgenese von Czernowitz mit Blick auf die sozialtopographischen Veränderungen, in: Gunda Barth-Scalmani/Kurt Scharr (Hrsg.), Die 
Gegenwart des Vergangenen im urbanen Raum Czernowitz-Innsbruck. Projektergebnisse eines gemeinsamen Studierendenprogrammes der Universitäten Czernowitz und Innsbruck über das kulturelle Erbe im öffentlichen Raum, Innsbruck 2019, S. 15-30.

Maner, Hans-Christian, Moldau, in: Online-Lexikon zur Kultur und Geschichte der Deutschen im östlichen Europa, 2013, [ome-lexikon.uni-oldenburg.de/54141.html], eingesehen 03.04.2020.

Mick, Christoph, Reise nach „Halb-Asien". Galizien als binnenexotisches Reiseziel, in: Peter Stachel/Martina Thomsen (Hrsg.), Zwischen Exotik und Vertrautem. Zum Tourismus in der Habsburgermonarchie und ihren Nachfolgestaaten, Bielefeld 2014, S. 95-112.

Österreichisches Biographisches Lexikon, Franzos Karl Emil, o. D., [https://www.biographien.ac.at/oebl/oebl_F/Franzos_Karl-Emil_1848_1904.xml;internal\&action=hilite. action\&Parameter=franzos* ${ }^{*}$, eingesehen 5.8.2019.

Österreichisches Biographisches Lexikon, Tomaszczuk (Tomaszuk) Konstantin (Constantin), o. D., [https://www.biographien.ac.at/oebl/oebl_T/Tomaszczuk_Konstantin_1840_1889.xml], eingesehen 04.02.2020.

Polek, Johann, Topographische Beschreibungen der Bukowina mit militärischen Anmerkungen von Major Friedrich von Mieg, in: Jahrbuch des Bukowiner Landesmuseums 5, Czernowitz 1897, S. 3-38.

Possar, P. A. Fédor K., Die russischen Ostsee-Provinzen Kurland, Livaland und Esthland, Stuttgart 1843

Rohrer, Joseph, Bemerkungen auf einer Reise von der türkischen Gränze über die Bukowina durch Ost- und Westgalizien, Schlesien und Mähren nach Wien, Wien 1804.

Rychlo, Peter, Czernowitz als geistige Lebensform. Die Stadt und ihre Kultur, in: Helmut Braun (Hrsg.), Die Geschichte einer untergegangenen Kulturmetropole, Berlin 2006, S. $7-30$.

Scharr, Kurt, Die Bukowina: Erkundungen einer Kulturlandschaft. Ein Reiseführer. WienKöln-Weimar 2007.

Ders., Die Landschaft Bukowina. Das Werden einer Region an der Peripherie 1774-1918, Wien-Köln-Weimar 2010.

Ders., Die spätneuzeitliche Siedlungstätigkeit in der Bukowina unter österreichischer Herrschaft 1774-1914. Formung einer Kulturlandschaft durch die Wechselwirkung zwischen Siedlung und dem Entstehen eines modernen Staates, in: 17. Innsbrucker Jahresbericht 2003-2007 der Innsbrucker Geographischen Gesellschaft, Innsbruck 2009, S. 60-77.

Schröer, K. J., Versuch einer Darstellung der deutschen Mundarten des ungarischen Berglandes mit Sprachproben und Erläuterungen, Wien 1864.

Wagner, Hans, Joseph II, in: Neue Deutsche Biographie, Bd. 10, Berlin 1974, S. 617-622.

Wandruszka, Adam, Leopold II, in: Neue Deutsche Biographie, Bd. 14, Berlin 1985, S. 260-266. 
Weczerka, Hugo, Die „Francisco-Josephina” in Czernowitz. Eine Universität am Ostrand der Habsburgermonarchie, in: Wolfgang Dahmen/Johannes Kramer/Victoria Popovici (Hrsg.), Gelebte Multikulturalität. Czernowitz und die Bukowina, Frankfurt am Main 2010, S. 67-86.

Alexander Renner ist Student der Geschichtswissenschaften an der Universität Innsbruck. alexander.renner@student.uibk.ac.at

\section{Zitation dieses Beitrages}

Alexander Renner, Die Bukowina als eine Insel des „Deutschthums“ im Osten? Deutsche Kulturverbreitung und deren Wahrnehmung in Reiseberichten aus dem 19. Jahrhundert, in: historia.scribere 12 (2020), S. 43-58, [http://historia.sribere.at], eingesehen 16.6.2020 (=aktuelles Datum).

(C) Creative Commons Licences 3.0 Österreich unter Wahrung der Urheberrechte der Autorlnnen. 